\title{
Review
}

\section{Progesterone receptors - animal models and cell signaling in breast cancer Progesterone's role in mammary gland development and tumorigenesis as disclosed by experimental mouse genetics}

\author{
Selma Soyal, Preeti M Ismail, Jie Li, Biserka Mulac-Jericevic, Orla M Conneely and John P Lydon
}

Department of Molecular and Cellular Biology, Baylor College of Medicine, Houston, Texas, USA

Correspondence: Dr John P Lydon, Department of Molecular and Cellular Biology, Baylor College of Medicine, One Baylor Plaza, Houston, TX 77030, USA. Tel: +1 713798 3534; fax: +1 713790 1275; e-mail: jlydon@bcm.tmc.edu

Received: 17 April 2002

Revisions requested: 13 May 2002

Revisions received: 24 May 2002

Accepted: 28 May 2002

Published: 5 July 2002
Breast Cancer Res 2002, 4:191-196

(C) 2002 BioMed Central Ltd

(Print ISSN 1465-5411; Online ISSN 1465-542X)

\begin{abstract}
The progesterone receptor knockout mouse demonstrated progesterone's importance to parityinduced mammary tertiary branching and lobuloalveologenesis. Because early parity provides significant protection against breast cancer whereas prolonged exposure to premenopausal ovarian progesterone (or to postmenopausal supplementations thereof) has been linked to breast cancer risk, this steroid can be considered to exhibit contrasting roles in breast cancer etiology. This review describes the important mouse models that have contributed to our understanding of progesterone's role in mammary gland development and neoplasia. We conclude by emphasising the urgent need to identify the molecular targets of the progesterone receptor, and to determine whether these targets are modulated differently by the progesterone receptor isoforms ( $A$ and $B$ ) during mammary morphogenesis and tumorigenesis.
\end{abstract}

Keywords: knockout, mammary gland, progesterone, progesterone receptor, tumorigenesis

\section{Introduction}

Epidemiological studies have shown that early onset of menarche, delayed entry into menopause, cycle periodicity, nulliparity, and a late first pregnancy represent individual risk factors for breast cancer. However, early menopause and early first parity decrease this risk [1-3]. Because progesterone's presence or absence directly influences the establishment of each of these reproductive endocrine states, assessing mammary gland development and tumorigenesis in relation to progesterone exposure is of paramount importance.

As for most epithelial cancers, the incidence of human breast cancer is age dependent, implicating a stochastic multistep progression in the development of this disease [4]. Although breast cancer incidence increases with age, the increase is not uniform but achieves its highest rate during the reproductive years of premenopause [3]. The data suggest that ovarian steroidal exposure of the mammary epithelial cell (MEC) during this reproductive period underlies the risk to breast cancer presented by many of the aforementioned reproductive endocrine states [3].

One hypothesis to account for the link between ovarian steroidal exposure and human breast cancer risk is that ovarian steroid-induced MEC proliferation provides a temporal window of opportunity for the progressive acquisition of genetic errors [3]. As a result of these errors, the transformed MEC is predicted to undergo unchecked clonal expansion to a mammary neoplasm.

$\mathrm{MEC}=$ mammary epithelial cell; PR = progesterone receptor; PRAKO = a specific knockout for the PR-A isoform; PRBKO = a specific knockout for the PR-B isoform; PRKO = progesterone receptor knockout. 
Although estrogen's proliferative effects on mammary gland development and tumorigenesis are well recognized, progesterone's contribution to these processes has been mired in controversy [5-7]. Much of this controversy has been exacerbated in the past by: first, our inability to mechanistically dissect the individual roles of estrogen and progesterone in vivo; second, progesterone's established inhibitory role on estrogen-induced luminal epithelial proliferation in the uterus, and the extrapolation of this inhibitory role to the mammary gland; and third, the increasing number of conflicting reports concerning the importance of synthetic progestins in mammary tumor progression in the rodent $[8,9]$.

The acknowledged gaps in our understanding concerning progesterone's involvement in human breast cancer has exposed the urgent need to better understand the role played by progesterone as an endocrine mammogen in normal mammary gland development.

In the present review, we provide an overview of how experimental mouse genetics has not only defined progesterone's unique contribution to mammary gland morphogenesis and function, but has also aided in the formulation of new concepts concerning the role of this ovarian steroid in the normal and neoplastic development of this tissue.

\section{Postnatal mammary gland development The mouse as an experimental tool of inquiry}

Because many of the fundamental aspects of mammary gland development and tumorigenesis are conserved between rodents and humans [10], the rodent model (in particular, the rat and the mouse) has historically served as the experimental system of choice for in vivo mammary gland research. With the recent advent of powerful genetic approaches to manipulate the murine genome, the mouse offers an unprecedented level of sophistication with which to query systemic or locally acting hormonal controls on mammogenesis, from the genetic, cellular, and molecular perspectives.

As with the human, murine postnatal mammary gland development consists of two distinct allometric growth stages. The first of these stages occurs at the onset of puberty, whereas the second manifests in response to pregnancy [11] (Fig. 1a). At puberty, in response to systemic estrogen and locally acting growth factors, cap cells of terminal end buds undergo extensive mitosis to drive ductal elongation and dichotomous branching to the limits of the fat pad. On reaching adulthood, the mammary gland is growth quiescent except for incipient side branching and alveolar budding (conspicuous in most strains, but not all), which develop over time as a result of the exposure of the tissue to cycling levels of
Figure 1
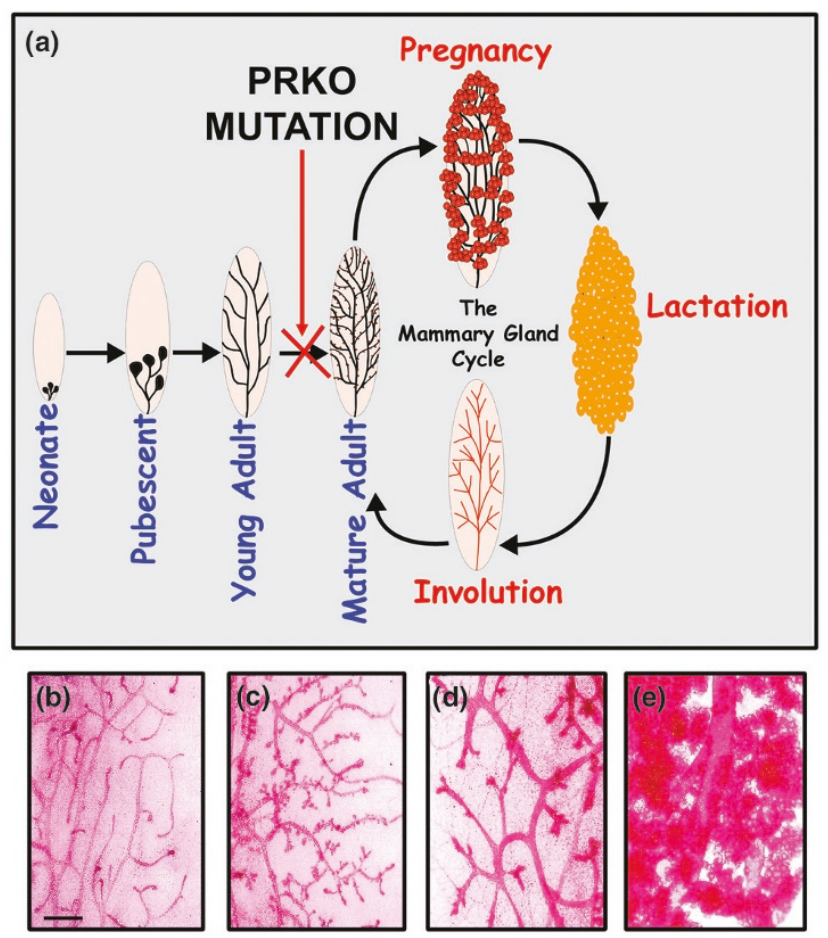

Progesterone receptor function is required for mammary ductal sidebranching and alveologenesis. (a) The salient postnatal stages of mammary gland development. Whole mounts of $(\mathbf{b})$ transplanted progesterone receptor knockout (PRKO) mammary glands and (c) wild-type mammary glands taken from a nulliparous host, and (d) transplanted PRKO mammary glands and (e) wild-type mammary glands taken from a parous host. Scale bar in (b) denotes $500 \mu \mathrm{m}$ and applies to all whole mounts. Adapted from Lydon et al. [5].

In response to the hormones of pregnancy, the epithelial compartment of the mammary gland undergoes proliferation and subsequent differentiation to generate alveoli that progressively fill the interductal spaces during late pregnancy, parturition, and lactation. Following weaning, removal of the suckling stimulus triggers collapse of the lobuloalveolar system through proteinase-mediated and apoptotic-mediated reductive remodeling processes, collectively termed involution. On completion of involution, the reinstatement of the mammary gland to a ductal architecture resembling the mammary phenotype of the prepregnant mouse completes the cycle of development (Fig. 1a).

Importantly, epidemiological and experimental investigations have provided irrefutable evidence that completion of this developmental cycle early in the reproductive life in rat, mouse and human species confers a significant protection against breast cancer in later life $[1,5]$. However, this protection is lost with a late first pregnancy. Given that the stage of mammary gland development at the time 
of carcinogen exposure greatly influences subsequent breast cancer initiation and progression, experimental mouse genetics has recently been applied to delineate progesterone's role in the elaboration of each of these developmental stages.

\section{The progesterone receptor knockout mouse The progesterone receptor}

Most of the physiological effects of progesterone are mediated by its intracellular receptor, the progesterone receptor (PR), which is a member of the nuclear receptor superfamily of transcription factors [12]. On binding progesterone ligand, the nuclear receptor ligand complex translocates to the nucleus to induce or to silence the expression of downstream target genes, which in turn manifest the physiological response of the target cell to the hormone, progesterone.

In most physiological systems, including the mammary gland, the PR is induced by estrogen, through its cognate nuclear receptor: the estrogen receptor. The PR comprises two isoforms, PR-A and PR-B, which are expressed from the same gene and are structurally identical except for a short amino acid extension contained in the $\mathrm{N}$-terminal region of $\mathrm{PR}-\mathrm{B}$. Previous transient transfection experiments demonstrated that the two PR isoforms exhibit distinct transactivational functions, which are dependent on the cell of origin and on the target gene promoter context [13]. Specifically, in cell types in which PR-A is inactive, the PR$B$ isoform (in the absence of PR-A) is a strong transactivator of several PR-regulated promoters [13].

In a physiological setting, these results suggest that the PR-A and PR-B isoforms may regulate distinct sets of target genes and may exhibit different transactivational capabilities in a given progesterone-responsive target tissue. Moreover, in cell and promoter contexts in which PR-A lacks transactivational activity, the coexpression of PR-A and PR-B demonstrated that the PR-A isoform could act as a dominant repressor of PR-B activity. In a cellular context in which PR-A and PR-B are coexpressed, this observation suggests that PR-A has the ability to attenuate the general progesterone responsiveness of specific PR target genes in specific target tissues in vivo.

\section{Characterization of the progesterone receptor knockout mouse}

To directly examine the physiological significance of $P R$ function in the murine mammary gland, a progesterone receptor knockout (PRKO) mouse model was generated in which both PR isoforms were simultaneously abrogated through gene targeting approaches [14]. Initial phenotypic characterization of the PRKO mouse revealed that removal of PR function resulted in a spectrum of reproductive abnormalities in the female, which included severe endocrine defects, an intrinsic impairment in ovulation, a dysfunctional uterus, and a loss in mating behaviors [14].
Unlike the estrogen receptor- $\alpha$ knockout mouse, in which the absence of estrogen signaling resulted in a block in mammary ductal outgrowth at puberty [15], the PRKO mouse mammary gland exhibited normal ductal elongation to generate a simple ductal architecture that was morphologically similar to the mammary gland of the young wildtype virgin [14]. Together, the estrogen receptor- $\alpha$ knockout and PRKO mouse models highlight the specific importance of ovarian estrogen rather than progesterone in epithelial ductal elongation in the pubescent mammary gland.

To address whether the progesterone signal is requisite for the observed increase in ductal side branching and lateral alveolar budding in the cycling nulliparous mouse, as well as for the observed morphological responses to the full spectrum of pregnancy hormones in the parous mouse, PRKO MECs were transplanted into the cleared mammary fat pad of a wild-type host [16]. The whole mammary gland transplantation approach was necessary to circumvent the inherent infertility defect of the PRKO mouse.

In the case of the cycling nulliparous host, the absence of ductal side branching and lateral alveolar budding in glands containing transplanted PRKO MECs (Fig. 1b) as compared with transplanted wild-type MECs (Fig. 1c) supports a role for progesterone in ovarian hormone-driven mammary epithelial cell proliferation. As indicated in the Introduction, ovarian steroidal-induced mammary epithelial proliferation has been implicated as a basis for nulliparityassociated breast cancer risk [3]. Indeed, the recent utilization of the PRKO mouse in combination with the chemical carcinogen-induced mammary tumor model demonstrated the critical role that progesterone-induced mammary epithelial proliferation can play in the initiation and progression of carcinogen-induced mammary tumors [17]. As a corollary to the aforementioned PRKO mammary tumor studies, recent investigations have indicated that progesterone may also facilitate chromosomal instability (aneuploidy) in the subsequent stages of mammary tumor progression that follow the loss of p53 function [18].

In the parous host, despite exposure to the hormonal milieu of pregnancy, transplanted PRKO MECs (Fig. 1d) failed to elicit further ductal side-branching and lobuloalveologenesis, as observed with transplanted wild-type MECs (Fig. 1e). Because early parity provides significant protection against breast cancer in later life, understanding the cellular and molecular mechanisms by which progesterone exerts these pregnancy-associated morphological and functional changes in the mammary gland is now an important priority for contemporary mammary gland research. To address this issue, recent studies have provided convincing evidence that progesterone (with estrogen) exerts a pivotal role in the elaboration of persistent molecular changes (i.e. the activation of p53-signaling 
pathways) in a subgroup of mammary epithelial cells that may contribute to parity-induced protection against mammary cancer [19]. Indeed, elegant studies recently reported by Wagner et al. [20] suggest that these parityinduced mammary epithelial populations may be identified and isolated in the near future.

\section{Future directions}

\section{A paracrine mode of action}

As in the human mammary gland [21], immunohistochemical studies on the murine mammary gland have demonstrated that PR expression is restricted to the luminal epithelial cell [22,23]. Importantly, recent immunofluorescence experiments have revealed that the majority of epithelial cells, which undergo proliferation in response to progesterone, are segregated from, but in close apposition to, PR-positive cells (Fig. 2a,b). These observations support a paracrine mode of action in which PR-positive cells (in response to progesterone) express and secrete a paracrine mediator(s) that impacts a neighboring PR-negative cell to proliferate (Fig. 2c). Interestingly, a paracrine mode of action for progesterone has also been shown to exist in the uterus [24]. Unlike the mammary gland, however, the progesterone-induced paracrine circuit emanates from the uterine stromal compartment to influence luminal epithelial proliferation.

The finding that the nonuniform cellular organization pattern for PR-positive cells and proliferating PR-negative cells is conserved between the human mammary gland and the rodent mammary gland $[21,25]$ has provided strong support for an evolutionary conserved cellular mechanism of action by which PR-positive cells influence neighboring PR-negative cells to participate in ductal morphogenesis.

Although the reasons for such an evolutionary conserved nonuniform cellular organization for PR expression is uncertain, derailment of such an important cellular arrangement is predicted to adversely affect normal mammary gland development. Indeed, in the case of many steroid receptor positive human breast tumors, the majority of estrogen and progesterone receptor positive tumor cells also undergo proliferation, clearly contravening the paracrine signaling circuit that exists in the normal gland $[21,25]$. Although speculative, the above studies suggest that the genesis of these tumors may require an early switch in steroid-dependent regulation of proliferation from a paracrine to an autocrine mechanism.

Additional support for the importance of this cellular organization pattern for PR expression to normal mammary gland development comes from the recent analysis of the adult CCAAT/enhancer binding protein $\beta$ knockout mammary gland, which does not respond to the prolifera-
Figure 2
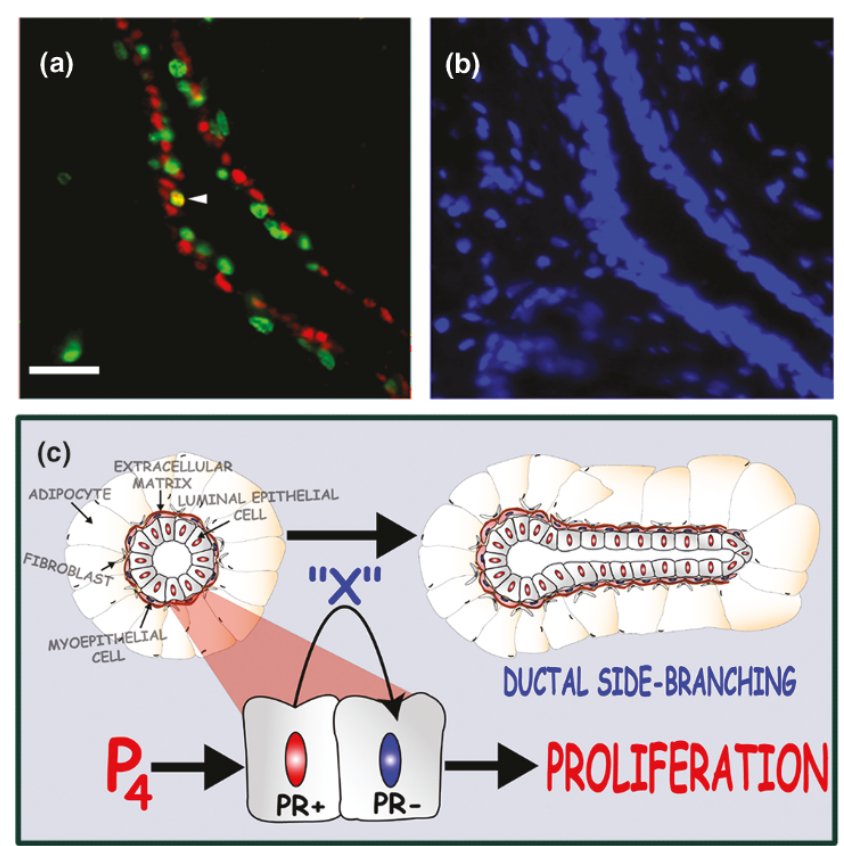

Paracrine mode of action for progesterone-induced branching morphogenesis in the mammary gland. (a) Indirect

immunofluorescence analysis of a rat mammary gland shows lumina epithelial cells expressing the progesterone receptor (PR) (red) or undergoing proliferation (green) as measured by 5-bromodeoxyuridine. The arrow indicates a single proliferating cell (yellow), which also expresses the PR. Scale bar represents $50 \mu \mathrm{m}$. (b) All nuclei in the field shown in (a), as detected by 6-diamidino-2-

phenylindole incorporation. Details of this experiment are described elsewhere [5]. (c) The conceptual idea of a paracrine mode of action for progesterone in mammary branching morphogenesis. ' $X$ ' denotes the elusive downstream paracrine mediator(s) of the progesterone (P4) proliferative signal. Adapted from Lydon et al. [5].

to a nonuniform cellular distribution pattern for PR expression observed in the normal gland, the CCAAT/enhancer binding protein $\beta$ knockout mammary gland exhibits a uniform cellular organization pattern for PR-positive cells with the attendant absence of PR-negative cells that proliferate in response to the progesterone paracrine signal.

Clearly, to gain a more meaningful mechanistic understanding of progesterone's paracrine mode of action in the mammary gland, identification of the downstream genetic networks that relay the progesterone signal will be essential. Towards this end, recent studies have provided strong evidence that the wnt-4 signaling pathway may act as an important paracrine mediator of the progesteroneinitiated proliferative signal in the mammary gland [26].

With the availability of the complete murine genome sequence, of gene discovery approaches, and of genetic strategies to conditionally turn on or turn off potential target genes in the murine mammary gland, we anticipate 
that progesterone's mammogenic effects will be readily exposed to molecular dissection in the near future.

\section{PR-A and PR-B}

Transgenic approaches have recently been employed to address whether the PR isoforms (PR-A and PR-B) mediate distinct physiological effects in the mammary gland $[27,28]$. Overexpression of PR-A resulted in increased ductal branching and eventual hyperplasia [27], whereas PR-B overexpression caused reduced ductal elongation and branching [28]. These in vivo investigations strongly support the concept that progesterone's physiological signal is interpreted differently by its two receptor isoforms, and that misexpression of either one of these isoforms can result in aberrant mammary gland development.

Using gene targeting approaches, a specific knockout for the PR-A isoform (PRAKO) has recently been generated and characterized [29]. The PRAKO mouse, like the PRKO mouse, exhibited a infertility phenotype. Unlike the PRKO mouse, however, the PRAKO mammary gland developed normally in the absence of PR-A function [29]. Although PR-A is expressed in the mammary gland, these studies suggest that, unlike most other progesterone target tissues, the PR-B isoform may functionally compensate for PR-A in the murine mammary gland.

To determine whether PR-B is indispensable for mammary development or whether its actions can be compensated by PR-A, a specific knockout for the PR-B isoform (PRBKO) mouse model has recently been generated and is currently being characterized. Unlike the PRAKO mouse, the PRBKO female is fertile. Initial mammary gland studies, however, indicate that loss of PR-B function results in reduced pregnancy-associated ductal branching compared with wild-type mammary glands (Conneely OM, unpublished data, 2002). These data, although preliminary, suggest that most of the mammogenic effects of progesterone are mediated by the PR-B isoform in the mouse.

\section{Conclusions}

Experimental mouse genetics has highlighted progesterone's indispensable role in pregnancy-induced morphological and functional changes in the mammary gland. If the aforementioned developmental changes occur early in reproductive life, a significant protection against breast cancer can be achieved. Because prolonged progesterone exposure, either through uninterrupted cyclical ovarian activity or by postmenopausal supplementation, has been linked to breast cancer risk, progesterone can be viewed as an endocrine mammogen with contrasting roles with respect to breast cancer etiology. With this view in mind, our concepts of breast cancer risk in relation to progesterone exposure will remain just that until the downstream genetic pathways through which progesterone affects normal and neoplastic mammary development are identified. With the availability of PRKO mouse models for PR-A, PR-B or both isoforms, in combination with DNA microarray approaches, we believe this goal will soon be realized.

\section{Acknowledgements}

The authors thank Dr Daniel Medina for critical reading of the manuscript. This work was supported in part by NIH grant CA-77530 and by DOD grant DAMD17-01-1-0138 to JPL.

\section{References}

1. MacMahon B, Cole P, Lin TM, Lowe CR, Mirra AP, Ravnihar B, Salber EJ, Valaoras VG, Yuasa S: Age at first birth and breast cancer risk. Bull World Health Org 1970, 43:209-221.

2. Kelsey JL, Gammon MD, John EM: Reproductive factors and breast cancer. Epidemiol Rev 1993, 15:36-47.

3. Spicer DV, Krecker EA, Pike MC: The endocrine prevention of breast cancer. Cancer Invest 1995, 13:495-504.

4. Loeb LA: A mutator phenotype in cancer. Cancer Res 2001, 61:3230-3239.

5. Lydon JP, Sivaraman L, Conneely OM: A reappraisal of progesterone action in the mammary gland. J Mammary Gland Biol Neoplasia 2000, 5:325-338.

6. Ross RK, Paganini-Hill A, Wan PC, Pike MC: Effect of hormone replacement therapy on breast cancer risk: estrogen versus estrogen plus progestin. J Natl Cancer Inst 2000, 92:328-332.

7. Sitruk-Ware R: Progestogens in hormal replacement therapy: new molecules, risks, and benefits. Menopause: J North Am Menopause Soc 2002, 9:6-15.

8. Li S, Leveesque C, Geng C-S, Yan X, Labrie F: Inhibitory effects of medroxyprogesterone acetate (MPA) and the pure antiestrogen EM-219 on estrone (E1)-stimulated growth of dimethylbenz(a)anthracene (DMBA)-induced mammary carcinoma in the rat. Breast Cancer Res Treat 1995, 34:147-159.

9. Aldaz CM, Liao QY, La Bate M, Johnston DA: Medroxyprogesterone acetate accelerates the development and increases the incidence of mouse mammary tumors induced by dimethylbenzanthracene. Carcinogenesis 1996, 17:20692072.

10. Cardiff RD: Validity of mouse mammary tumour models for human breast cancer: comparative pathology. Microsc Res Tech 2001, 52:224-230.

11. Silberstein GB: Postnatal mammary gland morphogenesis. Microsc Res Tech 2001, 52:155-162.

12. Tsai M-J, O'Malley BW: Molecular mechanisms of action of steroid/thyroid receptor superfamily members. Ann Rev Biochem 1994, 63:451-486.

13. Vegeto E, Shahbaz MM, Wen DX, Goldman ME, O'Malley BW, McDonnell DP: Human progesterone receptor A form is a cell and promoter specific repressor of human progesterone receptor B function. Mol Endocrinol 1993, 7:1244-1255.

14. Lydon JP, DeMayo FJ, Funk CR, Mani SK, Hughes AR, Montgomery CA Jr, Shyamala G, Conneely OM, O'Malley BW: Mice lacking progesterone receptors exhibit pleiotropic reproductive abnormalities. Genes Dev 1995, 9:2266-2278.

15. Bocchinfuso WP, Korach KS: Mammary gland development and tumorigenesis in estrogen receptor knockout mice. J Mammary Gland Biol Neoplasia 1997, 2:323-334.

16. Brisken C, Park S, Vass T, Lydon JP, O'Malley BW, Weinberg RA: A paracrine role for the epithelial progesterone receptor in mammary gland development. Proc Natl Acad Sci USA 1998, 95:5076-5081.

17. Lydon JP, Ge G, Kittrell FS, Medina D, O'Malley BW: Murine mammary gland carcinogenesis is critically dependent on progesterone receptor function. Cancer Res 1999, 59:42764284.

18. Goepfert TM, McCarthy M, Kittrell FS, Stephens C, Ullrich RL, Brinkley BR, Medina D: Progesterone facilitates chromosome instability (aneuploidy) in p53 null normal mammary epithelial cells. FASEB J 2000, 14:2221-2229.

19. Sivaraman L, Conneely OM, Medina D, O'Malley BW: p53 is a potential mediator of pregnancy and hormone-induced resistance to mammary carcinogenesis. Proc Natl Acad Sci USA 2001, 98:12379-12384. 
20. Wagner KU, Boulanger CA, Henry MD, Sgagias M, Hennighausen $\mathrm{L}$, Smith $\mathrm{GH}$ : An adjunct mammary epithelial cell population in parous females: its role in functional adaptation and tissue renewal. Development 2002, 129:1377-1386.

21. Clarke RB, Howell A, Potten CS, Anderson E: Dissociation between steroid receptor expression and cell proliferation in the human breast. Cancer Res 1997, 57:4987-4991.

22. Silberstein GB, Van Horn K, Shyamala G, Daniel CW: Progesterone receptors in the mouse mammary duct: distribution and developmental regulation. Cell Growth Differ 1996, 7:945952.

23. Seagroves TN, Lydon JP, Hovey RC, Vonderhaar BK, Rosen JM: $\mathrm{C} / \mathrm{EBPb}$ (CCAAT/Enhancer Binding Protein) controls cell fate determination during mammary gland development. $\mathrm{Mol}$ Endocrinol 20001, 4:359-368.

24. Kurita T, Young P, Brody JR, Lydon JP, O'Malley, Cunha GR: Stromal progesterone receptors mediate the inhibitory effects of progesterone on estrogen induced uterine epithelial cell deoxyribonucleic acid synthesis. Endocrinology 1999, 139:4708-4713.

25. Clarke RB, Howell A, Potten CS, Anderson E: P27(KIP1) expression indicates that steroid receptor-positive cells are a nonproliferating, differentiated subpopulation of the normal human breast epithelium. Eur J Cancer 2000, 36(suppl 4): S28-S29.

26. Brisken C, Heineman A, Chavarria T, Elenbaas B, Tan J, Dey SK, McMahon JA, McMahon AP, Weinberg RA: Essential function of Wnt-4 in mammary gland development downstream of progesterone signaling. Genes Dev 2000, 14:650-654.

27. Shyamala G, Yang $X$, Silberstein G, Barcellos-Hoff MH, Dale E: Transgenic mice carrying an imbalance in the native-ratio of $A$ to $B$ forms of progesterone receptor exhibit developmental abnormalities in mammary glands. Proc Natl Acad Sci USA 1998, 95:696-701.

28. Shyamala G, Yang X, Cardiff RD, Dale E: Impact of progesterone receptor on cell-fate decisions during mammary gland development. Proc Natl Acad Sci USA 2000, 97:3044-3049.

29. Mulac-Jericevic B, Mullinax RA, DeMayo FJ, Lydon JP, Conneely OM: Subgroup of reproductive functions of progesterone mediated by progesterone receptor-B isoform. Science 2000, 289:1751-1754. 